\title{
Cansancio del rol de cuidador principal del paciente en hemodiálisis
}

\author{
Inmaculada Gil Barrera, Juan Antonio Pilares Puertas, Rubén Romero López, Modesta Santos Seco
}

\author{
Hospital Universitario Puerta del Mar. Cádiz
}

\section{Objetivo:}

El objetivo principal es conocer la prevalencia del diagnóstico enfermero: cansancio del rol del cuidador de los pacientes que realizan tratamiento de hemodiálisis. Se relacionará la sobrecarga del cuidador con el nivel de dependencia del paciente y se valorará el grado de conocimiento que posee éste sobre la enfermedad. Finalmente se relacionará el grado de sobrecarga con la presencia de cuadros de fatiga y/o síntomas depresivos.

\section{Metodología:}

Es un estudio descriptivo transversal en una muestra con 48cuidadores de pacientes crónicos. Las variables estudiadas fueron sexo, edad, relación parentesco y lugar de residencia del cuidador y del paciente; nivel de estudios y trabajo del cuidador. Al grupo de cuidadores se le aplicaron los siguientes instrumentos validados: Test de Zarit, Cuestionario de salud general de Goldberg y un Cuestionario de evaluación de conocimientos generales sobre diálisis; al grupo de pacientes se le aplicó el Indice de Barthel. Posteriormente se procedió a la tabulación y el análisis descriptivo de los datos.

\section{Resultados:}

La edad media de la muestra del grupo de pacientes fue de 67años y del grupo cuidadores de 60. La distribución de sexos correspondió mayoritariamente en el grupo de pacientes a hombres y en el de cuidadores a mujeres. En relación con el parentesco entre el cuidador y el paciente la mayor parte se corresponde con el cónyuge. Respecto al nivel de estudios, encontramos hasta un $63 \%$ con estudios primarios o sin estudios. El $79 \%$ de los cuidadores no trabaja y residen en la misma vivienda que el paciente. El test de Zarit refiere que el $45 \%$ de los cuidadores sienten una sobrecarga leve o moderada. El cuestionario de salud general de Goldberg revela que el $52 \%$ de los cuidadores padecen síntomas somáticos y ansiedad e insomnio; no revela una disfunción social y el $76 \%$ de los encuestados presentan síntomas depresivos. Según el Índice de Barthel la gran mayoría de los pacientes tienen una dependencia severa a moderada. El $71 \%$ de los cuidadores carecen de conocimientos generales sobre la diálisis, cuidados de accesos vasculares, alimentación 0 administración de medicación domiciliaria.

\section{Discusión y conclusiones:}

Comprobamos que el perfil del cuidador principal son mujeres con una edad media de 60 años, con un nivel de estudios bajo y que no trabaja fuera del domicilio. No identifican verbalmente el cansancio acumulado pero se comprueba la dependencia severa a moderada de este tipo de pacientes que provoca la presencia de síntomas somáticos, ansiedad e insomnio y en la gran mayoría síntomas depresivos. Cuanto mayor es la dependencia se agudizan más estos síntomas. También se comprueba una falta de conocimientos generales sobre la enfermedad y sus cuidados por lo que su formación podría disminuir la sobrecarga llevada y sus consecuencias, el personal de la unidad comenzará brevemente talleres para paliar esta falta de conocimientos donde se enfocará la nueva forma de vida que conlleva esta enfermedad transmitiendo seguridad, tranquilidad y al mismo tiempo nuevos conocimientos. 


\section{Referencias Bibliográficas}

1. Andreu L, Moreno C, Julve M. Características del cuidado familiar a pacientes dependientes en programa de hemodiálisis. Rev Soc Esp Enferm Nefrol 2011;14(1):51/52.

2. Cousineau N, McDowell I, Hotz S, Hébert P. Measuring Chronic Patients Feelings of Being a Burden to their Caregivers. Medical Care 2003; volume 41, number1: 110-118.

3. Teixido J, Tarrats L, Arias N, Cosculluela. Cuestionario de sobrecarga de cuidadores de pacientes de diálisis peritoneal. Rev Nefrología 2006; volumen 26, número 1:74-83. 